\title{
Learning Driver Behaviors Using A Gaussian Process Augmented State-Space Model
}

Anton Kullberg, Isaac Skog and Gustaf Hendeby

The self-archived postprint version of this journal article is available at Linköping University Institutional Repository (DiVA):

http:/ / urn.kb.se/ resolve?urn=urn:nbn:se:liu:diva-167484

N.B.: When citing this work, cite the original publication.

Kullberg, A., Skog, I., Hendeby, G., (2020), Learning Driver Behaviors Using A Gaussian Process Augmented State-Space Model, Proceedings of 2020 23rd International Conference on Information Fusion (FUSION 2020), 530-536. https:// doi.org/ 10.23919/ FUSION45008.2020.9190245

Original publication available at:

https:/ / doi.org/ 10.23919/ FUSION45008.2020.9190245

Copyright: Institute of Electrical and Electronics Engineers (IEEE)

http:// www.ieee.org/

(C) 2020 IEEE. Personal use of this material is permitted. However, permission to reprint/ republish this material for advertising or promotional purposes or for creating new collective works for resale or redistribution to servers or lists, or to reuse any copyrighted component of this work in other works must be obtained from the IEEE. 


\section{Learning Driver Behaviors Using A Gaussian Process Augmented State-Space Model}

\author{
Anton Kullberg \\ Linköping University \\ anton.kullberg@liu.se
}

\author{
Isaac Skog \\ Linköping University \\ isaac.skog@liu.se
}

\author{
Gustaf Hendeby \\ Linköping University \\ gustaf.hendeby@liu.se
}

\begin{abstract}
An inference method for Gaussian process augmented state-space models are presented. This class of greybox models enables domain knowledge to be incorporated in the inference process to guarantee a minimum of performance, still they are flexible enough to permit learning of partially unknown model dynamics and inputs. To facilitate online (recursive) inference of the model a sparse approximation of the Gaussian process based upon inducing points is presented. To illustrate the application of the model and the inference method, an example where it is used to track the position and learn the behavior of a set of cars passing through an intersection, is presented. Compared to the case when only the state-space model is used, the use of the augmented state-space model gives both a reduced estimation error and bias.
\end{abstract}

\section{INTRODUCTION}

State estimation is a fundamental problem in many areas, such as robotics, target tracking, economics, etc. Typical techniques for state estimation are Bayesian filters such as the Kalman Filter (KF), Extended Kalman Filter (EKF) or the Particle Filter $(\mathrm{PF})$ [1]. Common to these techniques is that they require a model for the state dynamics as well as an observation model for the considered system. A general model for this is

$$
\begin{aligned}
& \mathbf{x}_{k}=\mathbf{f}_{k}\left(\mathbf{x}_{k-1}, \mathbf{u}_{k}, \mathbf{w}_{k}\right) \\
& \mathbf{y}_{k}=\mathbf{h}_{k}\left(\mathbf{x}_{k}, \mathbf{e}_{k}\right),
\end{aligned}
$$

where $\mathbf{f}_{k}$ and $\mathbf{h}_{k}$ are the state dynamics and observation model, respectively, and can be both time-varying and nonlinear. Further, $\mathbf{x}_{k}$ is the state vector at time $k, \mathbf{u}_{k}$ is some input, and $\mathbf{w}_{k}$ and $\mathbf{e}_{k}$ are mutually independent Gaussian white noise with covariance matrices $Q$ and $R$, respectively. Commonly, $\mathbf{f}_{k}$ and $\mathbf{h}_{k}$ are chosen based upon insight about the underlying physical process and the sensors used to observe it [2].

If information about the underlying process is missing, a model can be inferred from data using system identification methods [2]. This can for instance be done using a Gaussian Process State-Space Model (GPSSM), where both the state dynamics and observation model are viewed as separate Gaussian Processes (GPs) and learned jointly [3]-[7]. These methods may include prior physical knowledge, but only through the prior mean and is thus not so flexible.

\section{A. Augmented State-Space Models}

In many situations, knowledge about parts of the underlying process might exist, or a simplified model of the process exists. One way to incorporate this knowledge into the state estimation problem is to separate the system into two parts, one known (here assumed linear) and one unknown part, where the unknown part is modeled as a GP [8]. That is,

$$
\begin{aligned}
& \mathbf{f}_{k}\left(\mathbf{x}_{k-1}, \mathbf{u}_{k}, \mathbf{w}_{k}\right) \simeq F_{k} \mathbf{x}_{k-1}+G_{k} \mathbf{u}_{k}+G_{k} \mathbf{w}_{k} \\
& \mathbf{u}_{k}=\left[\begin{array}{llll}
u^{1}\left(\mathbf{z}_{k}\right) & u^{2}\left(\mathbf{z}_{k}\right) & \cdots & u^{J}\left(\mathbf{z}_{k}\right)
\end{array}\right]^{T} \\
& u^{j}(\mathbf{z}) \sim \mathcal{G P}\left(0, \kappa\left(\mathbf{z}, \mathbf{z}^{\prime}\right)\right) .
\end{aligned}
$$

$F_{k}$ is the state transition matrix and $G_{k}$ the input and noise matrix, where subscript $k$ refers to time, i.e., they may be time-varying. Furthermore, $\mathbf{u}_{k}$ is an unknown input at time $k$, which is to be inferred from data. It depends on some variable $\mathbf{z}_{k}$, which may change over time. For example, $\mathbf{z}_{k}$ could be a function of the state $\mathbf{x}_{k}$. Furthermore, the input $\mathbf{u}_{k}$ is modeled as a GP but could also, e.g., be a basis function expansion or neural network [9], [10]. Henceforth, the model (2) will be referred to as a Gaussian Process Augmented StateSpace Model (GPASSM). The benefits of this class of grey-box models are that they provide:

1) Performance guarantees - the model will perform at least as well as the simplified state dynamics model as the GP reverts to its prior outside the GP input domain.

2) Interpretability of $\mathbf{u}$ - in many cases, the learned inputs have a meaningful, physical interpretation.

3) Improvement over time - as more data is available, the model accuracy improves and so also the state estimation accuracy.

4) Information sharing - if many systems behave similarly, the input $\mathbf{u}$ may be shared between systems and learned jointly.

This class of models could for instance be exploited for tracking ships, or icebergs, where the learned input could be interpreted as the ocean currents [8]. It could also be used for estimating the structural integrity of buildings which is affected by unknown outer forces, which could be learned [11]. A final example is predicting the number of remaining charging cycles of batteries. In Lithium-Ion batteries it is well understood that the usage history and environment affect the degradation process [12]. These effects can potentially be learned from other batteries with similar history to accurately predict degradation and schedule maintenance accordingly. 


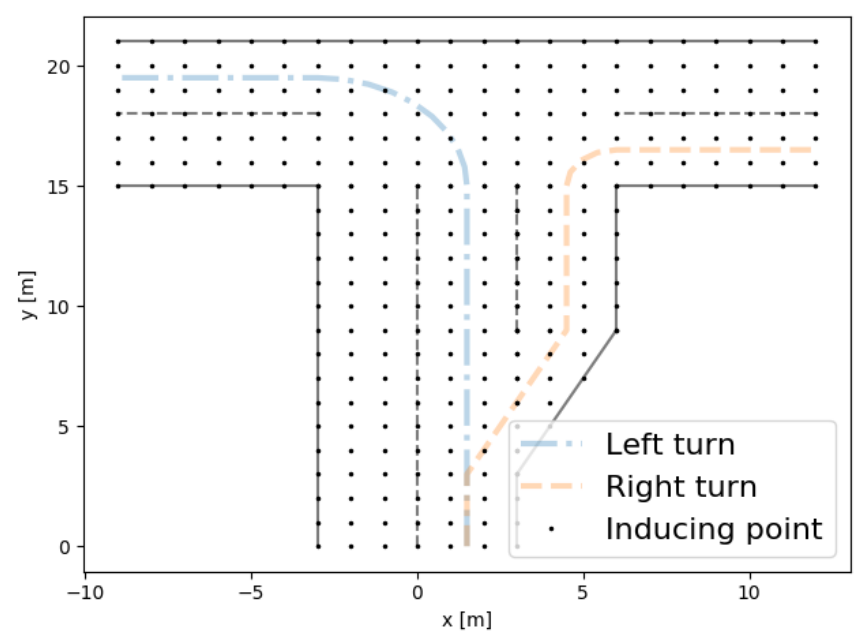

Fig. 1: Considered intersection scenario. A vehicle approaches from the middle road and may, with equal probability, travel along either of the two paths, i.e., either turn left or right. The inducing points are fixed on a grid covering the entire intersection.

\section{B. Scenario Description}

Before an estimator for the GPASSM is derived, a usage scenario is presented to give a foundation for the forthcoming discussion. The considered scenario is visualized in Fig 1. A vehicle approaches the intersection from the middle road and travels through the intersection according to one of the two possible paths. The problem is to estimate the state of the vehicle given by

$$
\mathbf{x}_{k}=\left[\begin{array}{llll}
p_{x} & p_{y} & v_{x} & v_{y}
\end{array}\right]^{T},
$$

where $p_{i}$ and $v_{i}$ is the position and velocity along the $i$ :thaxis, respectively. In a typical vehicle tracking application, the information provided by its trajectory is forgotten and the trajectory of each new vehicle passing through the intersection is assumed independent from its predecessors. In scenarios such as that illustrated by Fig 1, this assumption is naive since each vehicle in the intersection are bound to follow traffic regulations, the road network, etc. Hence, there will be a high correlation between the dynamics of the vehicles that pass through the intersection. This dynamic can be modeled as an unknown input to the model of the vehicles' motion and can be learned by sharing information between vehicles. To that end, the motion dynamics of the vehicles will be modeled by a GPASSM where the known model dynamics is given by a Constant Velocity (CV) model [13], and the unknown driver input, i.e., the accelerations, are modeled by a GP.

\section{MODELING}

In its basic form, the GPASSM presented in (2) cannot be used for online learning and state estimation as the inference of the GP increases cubically with the number of observations [14]. Therefore, we will next introduce a sparse approximation for the Gaussian process and modify the model GPASSM accordingly. For simplicity, but without loss of generality, the observation function will be assumed linear. Should a nonlinear function be chosen instead, the measurement update described in Section III will be slightly altered.

\section{A. Approximating the input}

There has been a lot of research in approximating GP regression, both in the static cases where all of the data is available at training time, as well as in the dynamic case where the data is added over time. These approximations can be divided into two distinct tracks, inducing inputs and basis function expansion. A comprehensive review of inducing input approaches can be found in [15]. An introduction to basis function expansion can be found in [3], [14], [16], [17].

Here, each GP will be approximated using inducing inputs, which is a set of discrete points fixed at some arbitrary location. Here, the points are fixed at $\mathbf{z}_{l}^{\xi}$ for $l=1, \ldots, L$ and their corresponding function values are given by $\boldsymbol{\xi}_{l}=\left[\begin{array}{llll}\xi^{1}\left(\mathbf{z}_{l}^{\xi}\right) & \xi^{2}\left(\mathbf{z}_{l}^{\xi}\right) & \cdots & \xi^{J}\left(\mathbf{z}_{l}^{\xi}\right)\end{array}\right]^{T}$ and are referred to as inducing inputs. Gather the inducing inputs in the vector $\Xi=\left[\begin{array}{llll}\boldsymbol{\xi}_{1}^{T} & \boldsymbol{\xi}_{2}^{T} & \cdots & \boldsymbol{\xi}_{L}^{T}\end{array}\right]^{T}$ and their corresponding coordinates in $\mathcal{Z}^{\xi}=\left[\begin{array}{llll}\mathbf{z}_{1}^{\xi} & \mathbf{z}_{2}^{\xi} & \cdots & \mathbf{z}_{L}^{\xi}\end{array}\right]^{T}$. Next, let

$$
\begin{aligned}
& K_{\xi \xi}=K\left(\mathcal{Z}^{\xi}, \mathcal{Z}^{\xi}\right)=\left[\begin{array}{ccc}
\kappa\left(\mathbf{z}_{1}^{\xi}, \mathbf{z}_{1}^{\xi}\right) & \cdots & \kappa\left(\mathbf{z}_{1}^{\xi}, \mathbf{z}_{L}^{\xi}\right) \\
\vdots & \ddots & \vdots \\
\kappa\left(\mathbf{z}_{L}^{\xi}, \mathbf{z}_{1}^{\xi}\right) & \cdots & \kappa\left(\mathbf{z}_{L}^{\xi}, \mathbf{z}_{L}^{\xi}\right)
\end{array}\right] \text {, } \\
& K_{\cdot \xi}^{k}=K\left(\mathbf{z}_{k}, \mathcal{Z}^{\xi}\right)=\left[\begin{array}{lll}
\kappa\left(\mathbf{z}_{k}, \mathbf{z}_{1}^{\xi}\right) & \cdots & \kappa\left(\mathbf{z}_{k}, \mathbf{z}_{L}^{\xi}\right)
\end{array}\right], \\
& \widetilde{K}=K \otimes I \text {, }
\end{aligned}
$$

where $I$ is the identity matrix. If the inputs are assumed independent of each other and the same kernel is used for each input $\xi^{j}$, the complete covariance matrix for $\Xi$ is given by $\widetilde{K}_{\xi \xi}$. By using the Fully Independent Conditional (FIC) approximation [15], [18], the model can now be written as

$$
\begin{aligned}
\mathbf{x}_{k} & =F_{k} \mathbf{x}_{k-1}+G_{k}\left(\widetilde{K}_{\cdot \xi}^{k} \widetilde{K}_{\xi \xi}^{-1} \Xi+\mathbf{v}_{k}\right) \\
\mathbf{y}_{k} & =H_{k} \mathbf{x}_{k}+\mathbf{e}_{k} \\
\Xi & \sim \mathcal{N}\left(0, \widetilde{K}_{\xi \xi}\right)
\end{aligned}
$$

where $\mathbf{v}_{k}$ is Gaussian white noise with covariance matrix $\Lambda_{k}=\kappa\left(\mathbf{z}_{k}, \mathbf{z}_{k}\right)-K_{\cdot \xi}^{k} K_{\xi \xi}^{-1}\left[K_{\cdot \xi}^{k}\right]^{T}$. Hence, the input at a given point $\mathbf{z}_{k}$ is given by a linear combination of the inducing inputs. Lastly, the matrix $\widetilde{K}_{\xi \xi}$ is not always well conditioned so to avoid numerical issues define

$$
\Psi=\widetilde{K}_{\xi \xi}^{-1} \Xi=\left[\begin{array}{llll}
\boldsymbol{\psi}_{1}^{T} & \boldsymbol{\psi}_{2}^{T} & \cdots & \boldsymbol{\psi}_{L}^{T}
\end{array}\right]^{T}
$$

and note that

$$
\operatorname{Cov}[\Psi]=\operatorname{Cov}\left[\widetilde{K}_{\xi \xi}^{-1} \Xi\right]=\widetilde{K}_{\xi \xi}^{-1} \widetilde{K}_{\xi \xi} \widetilde{K}_{\xi \xi}^{-T}=\widetilde{K}_{\xi \xi}^{-1},
$$

which is only needed for interpreting the covariance of the estimates and is not necessary for implementing the estimation. To allow the model to adapt to changes in the input, the 
inducing point state will be modeled as a random walk process. That is,

$$
\Psi_{k}=\Psi_{k-1}+\mathbf{w}_{k}
$$

where $\dot{\mathbf{w}}_{k}$ is Gaussian white noise with covariance matrix $\Sigma$. The state vector is then augmented with $\Psi$ and the model becomes

$$
\begin{aligned}
\mathbf{x}_{k} & =F_{k} \mathbf{x}_{k-1}+G_{k}\left(\widetilde{K}_{\cdot \xi}^{k} \Psi_{k-1}+\mathbf{v}_{k}\right) \\
\Psi_{k} & =\Psi_{k-1}+\mathbf{w}_{k} \\
\mathbf{y}_{k} & =H_{k} \mathbf{x}_{k}+\mathbf{e}_{k},
\end{aligned}
$$

and note that $\widetilde{K}_{\cdot \xi}^{k}$ still depends on the parameters $\mathbf{z}$.

\section{B. State-dependent input}

In the considered scenario, the vehicle acceleration depends on the location of the vehicle within the intersection. Hence, the input depends on the position of the vehicle, i.e.,

$$
\begin{aligned}
\mathbf{z}_{k} & =D_{k} \mathbf{x}_{k-1} \\
D_{k} & =\left[\begin{array}{ll}
I & 0
\end{array}\right] .
\end{aligned}
$$

The acceleration of course also depends on the velocity of the vehicle (The centripetal acceleration through a curve is given by $a_{c}=v^{2} / R$, where $R$ is the radius of the curve and $v$ is the absolute speed.). However, as this would quadratically scale the inducing point space $\mathbf{z}_{l}^{\xi}$ (from $\mathbb{R}^{2}$ to $\mathbb{R}^{4}$ ), this is neglected

for computational reasons. The full model is then described by

$$
\begin{aligned}
\mathbf{x}_{k} & =F_{k} \mathbf{x}_{k-1}+G_{k}\left(\widetilde{K}_{\cdot \xi}^{k} \Psi_{k-1}+\mathbf{v}_{k}\right) \\
\Psi_{k} & =\Psi_{k-1}+\mathbf{w}_{k} \\
\mathbf{z}_{k} & =D_{k} \mathbf{x}_{k-1} \\
\mathbf{y}_{k} & =H_{k} \mathbf{x}_{k}+\mathbf{e}_{k} .
\end{aligned}
$$

Recall that $\widetilde{K}_{\cdot \xi}^{k}=K\left(\mathbf{z}_{k}, \mathcal{Z}^{\xi}\right) \otimes I$ and note that the model is now nonlinear in the states due to the dependence of $K\left(\mathbf{z}_{k}, \mathcal{Z}^{\xi}\right)$ on $\mathbf{x}_{k-1}$.

\section{Kernel specification}

The choice of kernel function specifies what family of functions the GP is able to approximate well [14]. A common choice is the squared exponential kernel

$$
\kappa\left(\mathbf{z}, \mathbf{z}^{*}\right)=\sigma_{f}^{2} \exp \left(-\frac{1}{2 l^{2}}\left\|\mathbf{z}-\mathbf{z}^{*}\right\|^{2}\right)
$$

which will be used here as well. The hyperparameters $\theta=$ $\left(\sigma_{f}^{2}, l\right)$ govern the properties of the kernel, where $\sigma_{f}^{2}$ controls the general variance and $l$ is the characteristic length-scale and controls the width of the kernel. The hyperparameters can either be learned online [19], [20] or selected manually based on insight about the physical properties of the input.

\section{ESTIMATION}

The model (12) is nonlinear in the states and can be recursively estimated using, e.g., an EKF based on a first order Taylor expansion [1]. The EKF assumes that the prediction and filter distributions are both normally distributed as

$$
\begin{aligned}
\left(\begin{array}{c}
\mathbf{x}_{k+1 \mid k} \\
\Psi_{k+1 \mid k}
\end{array}\right) & \sim \mathcal{N}\left(\left(\begin{array}{c}
\hat{\mathbf{x}}_{k+1 \mid k} \\
\hat{\Psi}_{k+1 \mid k}
\end{array}\right),\left(\begin{array}{ll}
\mathbf{P}_{k+1 \mid k}^{x} & \mathbf{P}_{k+1 \mid k}^{x \psi} \\
\mathbf{P}_{k+1 \mid k}^{\psi x} & \mathbf{P}_{k+1 \mid k}^{\psi}
\end{array}\right)\right) \\
\left(\begin{array}{c}
\mathbf{x}_{k \mid k} \\
\Psi_{k \mid k}
\end{array}\right) & \sim \mathcal{N}\left(\left(\begin{array}{c}
\hat{\mathbf{x}}_{k \mid k} \\
\hat{\Psi}_{k \mid k}
\end{array}\right),\left(\begin{array}{cc}
\mathbf{P}_{k \mid k}^{x} & \mathbf{P}_{k \mid k}^{x \psi} \\
\mathbf{P}_{k \mid k}^{\psi x} & \mathbf{P}_{k \mid k}^{\psi}
\end{array}\right)\right) .
\end{aligned}
$$

By using the upper triangular structure of the state transition model (12a) and (12b), the EKF time-update becomes [8]

$$
\begin{aligned}
\hat{\mathbf{x}}_{k+1 \mid k}= & F_{k} \hat{\mathbf{x}}_{k \mid k}+G_{k} \widetilde{K}_{\cdot \xi}\left(D_{k} \hat{\mathbf{x}}_{k \mid k}\right) \hat{\Psi}_{k \mid k} \\
\hat{\Psi}_{k+1 \mid k}= & \hat{\Psi}_{k \mid k} \\
P_{k+1 \mid k}^{x}= & \mathbf{F}_{x} P_{k \mid k}^{x} \mathbf{F}_{x}^{T}+\mathbf{F}_{x} P_{k \mid k}^{x \psi} \mathbf{F}_{\psi}^{T}+\mathbf{F}_{\psi} P_{k \mid k}^{\psi x} \mathbf{F}_{x}^{T} \\
& +\mathbf{F}_{\psi} P_{k \mid k}^{\psi} \mathbf{F}_{\psi}^{T}+G_{k} \Lambda_{k} G_{k}^{T} \\
P_{k+1 \mid k}^{x \psi}= & \mathbf{F}_{x} P_{k \mid k}^{x \psi}+\mathbf{F}_{\psi} P_{k \mid k}^{\psi} \\
P_{k+1 \mid k}^{\psi x}= & \left(P_{k+1 \mid k}^{x \psi}\right)^{T} \\
P_{k+1 \mid k}^{\psi}= & P_{k \mid k}^{\psi}+\Sigma
\end{aligned}
$$

and the measurement update becomes

$$
\begin{aligned}
S_{k} & \triangleq R+H_{k} P_{k \mid k-1}^{x} H_{k}^{T} \\
L_{k}^{x} & \triangleq P_{k \mid k-1}^{x} H_{k}^{T} S_{k}^{-1} \\
L_{k}^{\psi} & \triangleq P_{k \mid k-1}^{\psi x} H_{k}^{T} S_{k}^{-1} \\
\hat{\mathbf{x}}_{k \mid k} & =\hat{\mathbf{x}}_{k \mid k-1}+L_{k}^{x}\left(\mathbf{y}_{k}-H_{k} \hat{\mathbf{x}}_{k \mid k-1}\right) \\
\hat{\Psi}_{k \mid k} & =\hat{\Psi}_{k \mid k-1}+L_{k}^{\psi}\left(\mathbf{y}_{k}-H_{k} \hat{\mathbf{x}}_{k \mid k-1}\right) \\
P_{k \mid k}^{x} & =P_{k \mid k-1}^{x}-L_{k}^{x} S_{k}\left(L_{k}^{x}\right)^{T} \\
P_{k \mid k}^{x \psi} & =P_{k \mid k-1}^{x \psi}-L_{k}^{x} S_{k}\left(L_{k}^{\psi}\right)^{T} \\
P_{k \mid k}^{\psi x} & =\left(P_{k \mid k}^{x \psi}\right)^{T} \\
P_{k \mid k}^{\psi} & =P_{k \mid k-1}^{\psi}-L_{k}^{\psi} S_{k}\left(L_{k}^{\psi}\right)^{T}
\end{aligned}
$$

where

$$
\begin{aligned}
& \mathbf{F}_{x}=F_{k}-\frac{G_{k}}{l^{2}}\left(\sum_{l=1}^{L} k\left(\mathbf{z}_{k}, \mathbf{z}_{l}^{\xi}\right) \cdot\left(\mathbf{z}_{k}-\mathbf{z}_{l}^{\xi}\right)^{T} \boldsymbol{\psi}_{l}\right) D_{k} \\
& \mathbf{F}_{\psi}=G_{k} \widetilde{K}_{\cdot \xi}^{k}
\end{aligned}
$$

See Appendix A for a derivation.

\section{Simulation and Results}

To illustrate the application of the proposed estimation approach and the GPASSM, position observations from a set of vehicles passing through the intersection illustrated in Fig 1 were simulated. 
TABLE I: Simulation parameters for three-way intersection scenario

\begin{tabular}{c|c|c} 
Parameter & Description & Value \\
\hline $\mathrm{L}$ & \# Inducing points & 310 \\
$\mathrm{~N}$ & \# Vehicles & 30 \\
$f_{s}$ & Sampling rate & $2 \mathrm{~Hz}$ \\
$\sigma_{e}^{2}$ & Measurement noise variance & 0.2 \\
$\sigma_{f}^{2}$ & Kernel variance & 0.05 \\
$l$ & Kernel length scale & 0.5 \\
$\mathrm{R}$ & EKF measurement noise variance & 1 \\
$\delta_{\mathbf{z}_{l}^{\xi}}$ & Grid spacing & $1 \mathrm{~m}$
\end{tabular}

\section{A. Simulation Parameters}

There are a number of parameters to be either learned or chosen. Here, they are all manually selected based on prior knowledge of the physical properties of the scenario. For the vehicle motion, a CV is chosen, i.e.,

$$
F_{k}=\left[\begin{array}{cc}
1 & T \\
0 & 1
\end{array}\right] \otimes I \quad G_{k}=\left[\begin{array}{c}
T^{2} / 2 \\
T
\end{array}\right] \otimes I,
$$

where $T$ is the sampling interval. For the observation model, it is assumed that the position of the vehicle is measurable with some noise, i.e.,

$$
H_{k}=\left[\begin{array}{ll}
I & 0
\end{array}\right] \quad R=\sigma_{e}^{2} I,
$$

where $\sigma_{e}^{2}$ is the measurement noise variance.

As for the GP, there are three parameters to be chosen: the location of the inducing points $\mathbf{z}_{l}^{\xi}$, the kernel variance $\sigma_{f}^{2}$, and the kernel length scale $l$. The inducing points are fixed on a grid covering the entire intersection, see Fig 1, uniformly spaced using a grid spacing $\delta_{\mathbf{z}_{l}^{\xi}}=1 \mathrm{~m}$, which was chosen as a trade-off between accuracy and computational burden. The kernel variance and length scale are chosen under the notion that the acceleration of a vehicle is a local phenomena and varies quickly over distance/time. The length scale is thus chosen as $l=0.5$ and the variance as $\sigma_{f}^{2}=0.05$. The simulation parameters are summarized in Table I.

During the simulations, all the vehicles were initiated to the true initial position and velocity. In total, $M=100$ simulations were run. The estimated input is reused for each new vehicle, hence, enabling information sharing.

\section{B. Qualitative Analysis}

As a baseline, a CV model without the input learning was used. The Root Mean Squared Error (RMSE) was calculated for each vehicle in each separate simulation and is visualized in Fig 3. Fig 4 visualizes 50 trajectories of a $\mathrm{CV}$, as well as of the GPASSM where the input has been learned in advance using 30 vehicles. The state errors for the first vehicle on each path are given in Fig 5(a) and the last on each path in Fig 5(b). Note that this is not necessarily the first or last vehicle in total and also that there is no guarantee that the same number of vehicles have traversed each path.

From Fig 5(a) it is evident that already for the first vehicle, there are benefits of including the GP. Before any accelerations are experienced, the model mimics the $\mathrm{CV}$ model exactly, but

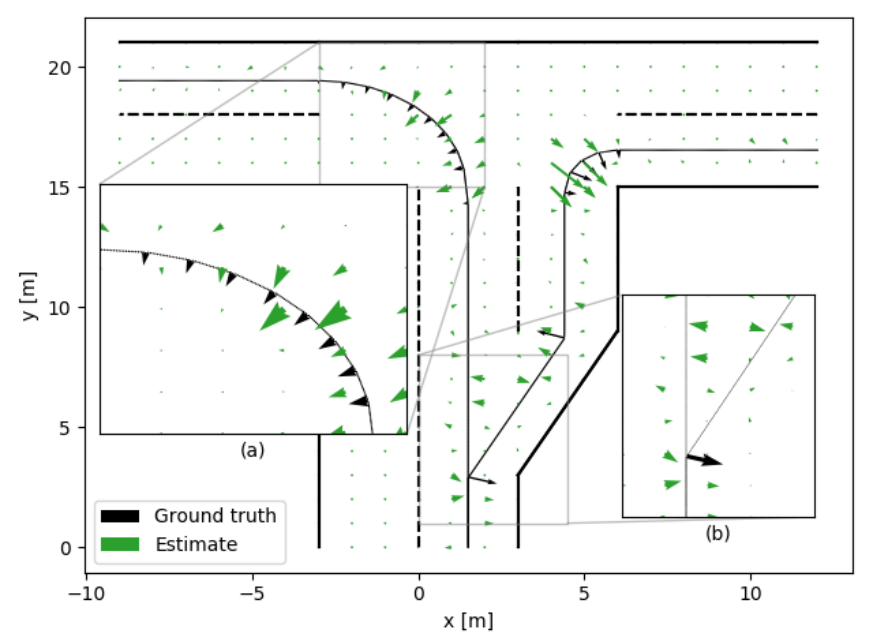

Fig. 2: Acceleration estimated by the GPASSM for one simulation with ground truth for comparison. Ground truth is plotted over the paths, estimates are over the sparse grid approximation. The estimated accelerations mimic the true accelerations well, see zoomed in area (a). At the path split point, see zoomed in area (b), the estimated accelerations diverge from the true.

as accelerations come into play (around time step 3-4) the proposed model improves over the standard CV. This is due to the process noise being inherently nonwhite, which the GP captures. As the number of vehicles increases, see Fig 5(b), the GPASSM learns the accelerations required to stay on the trajectory; see time step 15-25 for both paths. Lastly, even larger discrepancies between the GPASSM and CV model would be evident if $k$-step ahead prediction was used, since the CV would continue in a straight path and the GPASSM would follow the average trajectory of the past vehicles.

Now, there are some peculiarities. For instance, see Fig 5(b) where the GPASSM is actually worse than the $\mathrm{CV}$ model between time steps $3-10$. This is caused by the acceleration discontinuity where the two paths split. This is also evident in Fig 2, (zoomed in area (b)), where the discontinuity causes a lot of small sideways accelerations where the GP is compensating for its own errors in a sense. Fig 2 also indicates that the learned acceleration mimic the true closely, see zoomed in area (a).

From Fig 4 it is evident that the GPASSM follows the two paths better than the $\mathrm{CV}$ model. Whereas the $\mathrm{CV}$ model has a clear bias during the turns, the GPASSM does not suffer from this. However, given that the $\mathrm{CV}$ is a lot less computationally demanding, in a scenario where measurements are abundant and the sole interest is to estimate the position and velocity of a specific vehicle, this should still be the preferred choice. If fewer measurements are available, GPS spoofing is a real possibility, or information about common patterns is of interest, the GPASSM is to be preferred. 


\section{COnClusion And Future Work}

A Gaussian Process Augmented State-Space Model has been proposed for learning unknown, but common, accelerations of vehicles through static environments. The model generalizes to cases where a simple motion model is sought after, but where the bias associated with such are not. The model was shown to improve over an ordinary Constant Velocity (CV) model and removed the bias when the accelerations were non-zero. An issue with the model is that it can not handle ambiguities in the input it is trying to learn and will in some of these cases perform worse than a CV model. The model is, however, attractive as it allows a simple motion model to be used in combination with a data-driven model for learning unknown characteristics of the underlying system online. It also facilitates distributed learning of unknown characteristics between systems that behave similarly. The learned input function itself might also be of use since its physical interpretation in many cases is easily found.

For the model to reach its full potential, the input ambiguities must be addressed. It is also necessary to find an efficient way to factorize the input space so as to reduce the computational burden, e.g., through dividing the area of interest into hexagonal surfaces [17]. Moreover, the approximation strategy of the Gaussian Process needs to be evaluated. If an inducing input approach is used, methods to add, remove, or move these online is necessary for reducing computational burden and to enable the model to be used in large-scale applications.

\section{ACKNOWLEDGMENTS}

This work was partially supported by the Wallenberg AI, Autonomous Systems and Software Program (WASP) funded by the Knut and Alice Wallenberg Foundation.

The authors would also like to thank the reviewers for the valuable feedback, much of which will be addressed in future work.
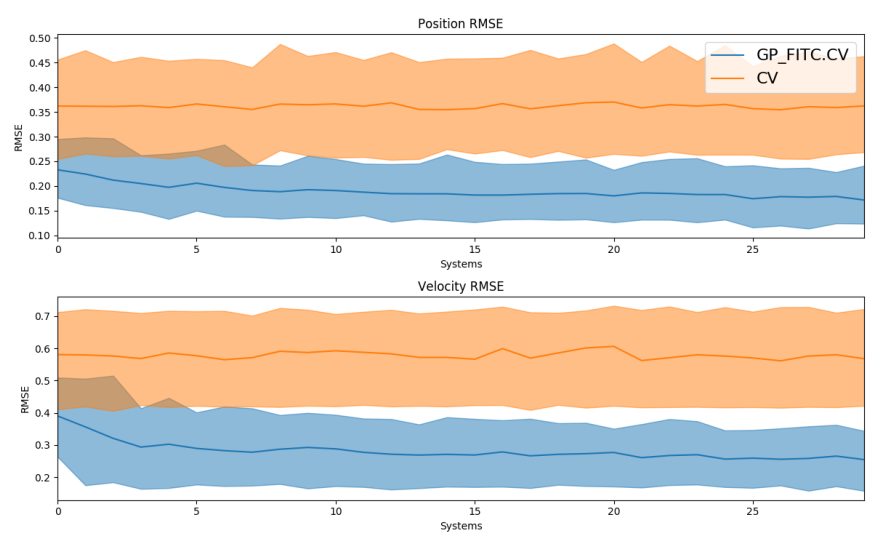

Fig. 3: RMSE over vehicles. Later vehicles use the accelerations learned from previous vehicles and thus give a lower RMSE for the GPASSM, but not for the CV model. Confidence bands are given by the 2.5 th and 97.5 th percentile over the simulations.

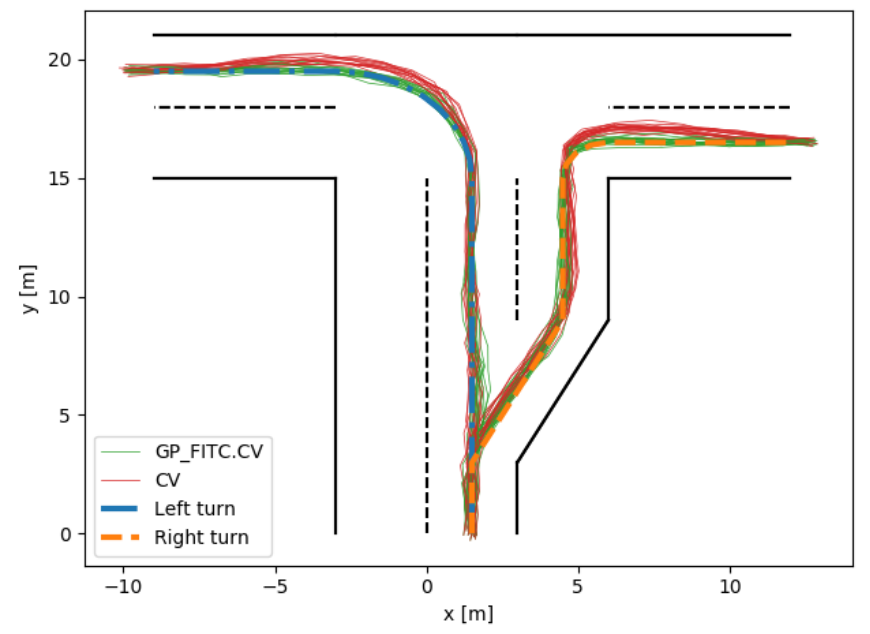

Fig. 4: 50 vehicle trajectories of the two models. The GPASSM is learned from a training data set of 30 vehicles. The $\mathrm{CV}$ model suffers from bias, which the GPASSM alleviates. That is, the green trajectories accurately follow the paths while the red do not. 


\section{APPENDIX A}

DERIVATION OF MODEL JACOBIAN

Given the model

$$
\begin{aligned}
\mathbf{x}_{k} & =F_{k} \mathbf{x}_{k-1}+G_{k}\left(\widetilde{K}_{\cdot \xi}^{k} \Psi_{k-1}+\mathbf{v}_{k}\right) \\
\Psi_{k} & =\Psi_{k-1}+\mathbf{w}_{k} \\
\mathbf{z}_{k} & =D \mathbf{x}_{k-1}
\end{aligned}
$$

the partial derivative of $\mathbf{x}_{k}$ with respect to $\mathbf{x}_{k-1}$ is given by

$$
\frac{\partial \mathbf{x}_{k}}{\partial \mathbf{x}_{k-1}}=F_{k}+G_{k}\left(\frac{\partial \widetilde{K}_{\cdot \xi}^{k}}{\partial \mathbf{x}_{k-1}} \Psi_{k-1}+\frac{\partial \mathbf{v}_{k}}{\partial \mathbf{x}_{k-1}}\right)
$$

where the derivative of the squared exponential kernel is given by

$$
\frac{\partial \kappa\left(\mathbf{z}, \mathbf{z}^{*}\right)}{\partial \mathbf{z}}=-\frac{\kappa\left(\mathbf{z}, \mathbf{z}^{*}\right)}{l^{2}}\left(\mathbf{z}-\mathbf{z}^{*}\right)^{T}
$$

and the derivative of the noise component $\mathbf{v}_{k}$ is given by

$$
\frac{\partial \mathbf{v}_{k}}{\partial \mathbf{x}_{k-1}}=\frac{\partial \mathbf{v}\left(D_{k} \mathbf{x}_{k-1}\right)}{\partial \mathbf{x}_{k-1}}=\frac{1}{2} \frac{\mathbf{v}_{k}}{\Lambda_{k}} \frac{\partial \Lambda_{k}}{\partial \mathbf{x}_{k-1}} D_{k}
$$

For proof, see Appendix B in [8]. Now, (A.2) can be written

$$
\begin{aligned}
\mathbf{F}_{x} \triangleq \frac{\partial \mathbf{x}_{k}}{\partial \mathbf{x}_{k-1}}= & F_{k}+G_{k}\left(-\frac{1}{l^{2}} \sum_{l=1}^{L}\left[\kappa\left(\mathbf{z}_{k}, \mathbf{z}_{l}^{\xi}\right)\left(\mathbf{z}_{k}-\mathbf{z}_{l}^{\xi}\right)^{T} \mathbf{w}_{l}\right]\right. \\
& \left.+\frac{1}{2} \frac{\mathbf{v}_{k}}{\Lambda_{k}} \frac{\partial \Lambda_{k}}{\partial \mathbf{x}_{k-1}}\right) D_{k}
\end{aligned}
$$

Furthermore the derivative of $\mathbf{x}_{k}$ w.r.t. $\Psi_{k-1}$ is given by

$$
\mathbf{F}_{\psi} \triangleq \frac{\partial \mathbf{x}_{k}}{\partial \Psi_{k-1}}=G_{k} \widetilde{K}_{\cdot \xi}^{k}
$$

\section{REFERENCES}

[1] S. Särkkä, Bayesian Filtering and Smoothing. Cambridge University Press, 2010.

[2] L. Ljung and T. Glad, Modeling and Identification of Dynamic Systems, 1 st ed. Studentlitteratur, 2016.

[3] A. Svensson, A. Solin, S. Särkkä, and T. B. Schön, "Computationally Efficient Bayesian Learning of Gaussian Process State Space Models," in AISTATS 2016, Cadiz, Spain, may 2016, pp. 213-221.

[4] A. Svensson and T. B. Schön, "A flexible state-space model for learning nonlinear dynamical systems," Automatica, 2017.

[5] R. Turner, M. P. Deisenroth, and C. E. Rasmussen, "State-space inference and learning with Gaussian processes," J. Mach. Learn. Res., vol. 9, pp. 868-875, 2010.

[6] R. Frigola, F. Lindsten, T. B. Schön, and C. E. Rasmussen, "Bayesian Inference and Learning in Gaussian Process State-Space Models with Particle MCMC," Adv. Neural Inf. Process. Syst., pp. 1-9, 2013.

[7] J. Ko and D. Fox, "GP-BayesFilters: Bayesian filtering using Gaussian process prediction and observation models," Auton. Robots, vol. 27, no. 1, pp. 75-90, 2009.

[8] C. Veibäck, J. Olofsson, T. R. Lauknes, and G. Hendeby, "Learning Target Dynamics While Tracking Using Gaussian Processes," IEEE Trans. Aerosp. Electron. Syst., vol. 9251, pp. 1-10, 2019.

[9] A. Svensson, T. B. Schön, A. Solin, and S. Särkkä, "Nonlinear State Space Model Identification Using a Regularized Basis Function Expansion," in CAMSAP 2015, no. Ml, Cancun, Mexico, dec 2015, pp 481-484.

[10] J. Sjöberg, H. Hjalmarsson, and L. Ljung, "Neural Networks in System Identification,” IFAC Proc. Vol., vol. 27, no. 8, pp. 359-382, 1994.

[11] R. Nayek, S. Chakraborty, and S. Narasimhan, "A Gaussian process latent force model for joint input-state estimation in linear structural systems," Mech. Syst. Signal Process., vol. 128, pp. 497-530, 2019.
[12] M. Ecker, N. Nieto, S. Käbitz, J. Schmalstieg, H. Blanke, A. Warnecke, and D. U. Sauer, "Calendar and cycle life study of $\mathrm{Li}(\mathrm{NiMnCo}) \mathrm{O} 2$-based 18650 lithium-ion batteries," J. Power Sources, vol. 248, pp. 839-851, 2014.

[13] X. R. Li and V. P. Jilkov, "Survey of Maneuvering Target Tracking. Part I: Dynamic Models," IEEE Trans. Aerosp. Electron. Syst., vol. 39, no. 4, pp. 1333-1364, 2003

[14] C. E. Rasmussen and C. K. I. Williams, Gaussian Processes for Machine Learning. MIT Press, 2006.

[15] J. Quiñonero-Candela and C. E. Rasmussen, "A Unifying View of Sparse Approximate Gaussian Process Regression," J. Mach. Learn. Res., vol. 6, pp. 1939-1959, 2005.

[16] A. Solin and S. Särkkä, "Hilbert space methods for reduced-rank Gaussian process regression," Stat. Comput., vol. 30, no. 2, pp. 419446, 2020.

[17] M. Kok and A. Solin, "Scalable Magnetic Field SLAM in 3D Using Gaussian Process Maps," in FUSION 2018, Cambridge, UK, jul 2018, pp. $1353-1360$.

[18] E. Snelson and Z. Ghahramani, "Sparse Gaussian Processes using Pseudo-inputs," Adv. Neural Inf. Process. Syst. 18, pp. 1257-1264, 2006.

[19] M. F. Huber, "Recursive Gaussian process: On-line regression and learning," Pattern Recognit. Lett., vol. 45, no. 1, pp. 85-91, 2014.

[20] M. K. Titsias, "Variational Learning of Inducing Variables in Sparse Gaussian Processes Michalis," in AISTATS 2009, D. van Dyk and M. Welling, Eds., vol. 5. Clearwater, Florida: JMLR, apr 2009, pp. $567-574$. 

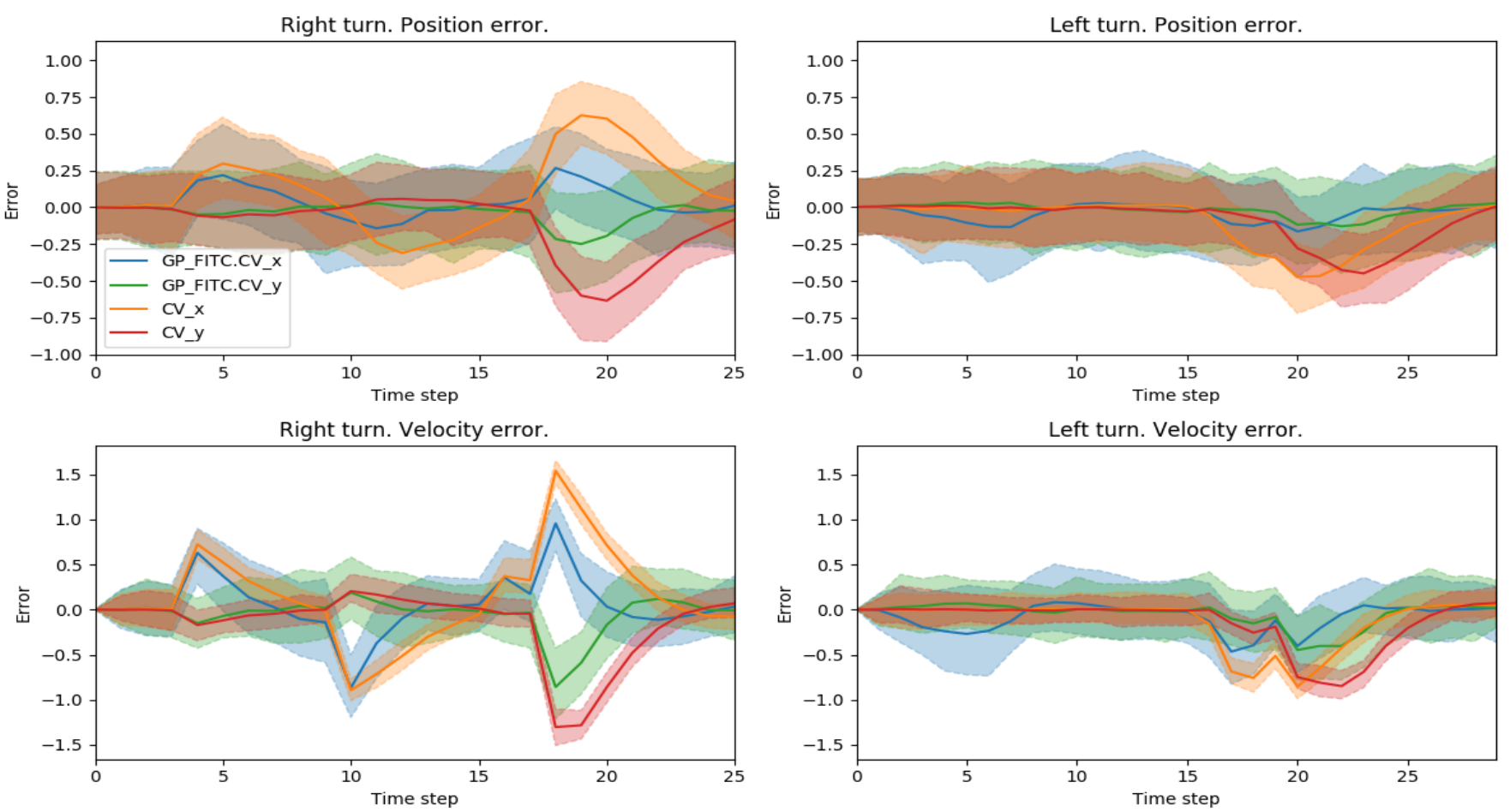

(a) The first vehicle on each path
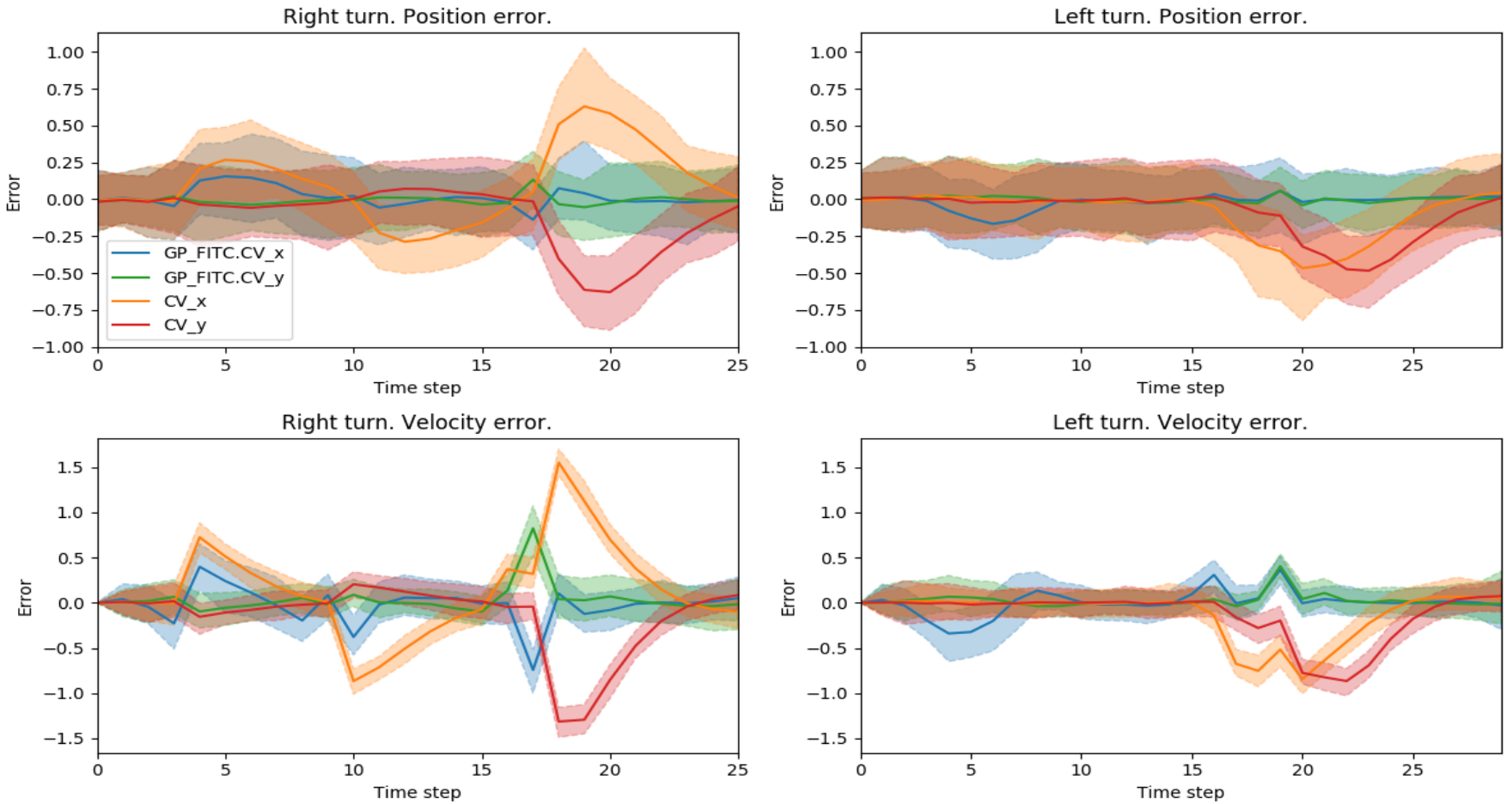

(b) The last vehicle on each path

Fig. 5: State errors for two different vehicles for both the GPASSM and CV model. Errors are separated by path and by dimension. Confidence bands are given by the 2.5th and 97.5 th percentile over the simulations. The two subfigures depict the first and the last vehicle on each path, i.e., it is not necessarily the first and last vehicle in total. 